\title{
ON THE DISTRIBUTION OF THE NONTRIVIAL ZEROS FOR THE DIRICHLET $L$-FUNCTIONS
}

\author{
XIAO-JUN YANG ${ }^{1,2,3}$
}

\begin{abstract}
This paper addresses a variant of the product for the Dirichlet $L^{-}$ functions. We propose a completely detailed proof for the truth of the generalized Riemann conjecture for the Dirichlet $L$-functions, which states that the real part of the nontrivial zeros is $1 / 2$. The Wang and Hardy-Littlewood theorems are also discussed with removing the need for it. The results are applicable to show the truth of the Goldbach's conjecture.
\end{abstract}

\section{Contents}

1. Introduction 1

2. Fundamental results 5

2.1. A variant of the product for the Riemann zeta function 5

2.2. A variant of the product for the Dirichlet $L$-functions 8

2.3. The Goldbach's problems 12

3. A detailed proof for the generalized Riemann conjecture 13

3.1. Family 1: $\chi=\chi_{0}$ and $q=1 \quad 13$

3.2. Family 2: $\chi=\chi_{0}$ and $q>1$

3.3. Family 3: $\chi=\chi^{*}$ and $\chi^{*}(-1)=1 \quad 16$

3.4. Family 4: $\chi=\chi^{*}$ and $\chi^{*}(-1)=-1 \quad 19$

4. New results and applications 22

4.1. New formulas for the Dirichlet $L$-functions 22

4.2. The truth of the Goldbach's conjecture 25

5. Conclusion 26

References $\quad 26$

1991 Mathematics Subject Classification. 11M06, 11M26.

Key words and phrases. Dirichlet $L$-function, generalized Riemann conjecture, nontrivial zeros, Goldbach's conjecture, Riemann zeta function. 


\section{IntRoduCtion}

The Dirichlet $L$-function $L(s, \chi)$ was formulated by German mathematician Johann Peter Gustav Lejeune Dirichlet in 1837 for the meromorphic continuation of the function defined by the series [1]

$$
L(s, \chi)=\sum_{n=1}^{\infty} \chi(n) s^{-n}
$$

where $\chi(n)$ is a Dirichlet character $(\bmod q>1), R e(s)>1, n \in \mathbb{N}$ and $s \in \mathbb{C}$. Here, $q$ is the prime, $\mathbb{N}$ and $\mathbb{C}$ are the sets of the natural numbers and complex numbers, and $\operatorname{Re}(s)=\sigma \in \mathbb{R}$ and $\operatorname{Im}(s)=t \in \mathbb{R}$ are the real and imaginary parts of the complex variable $s=\sigma+i t \in \mathbb{C}$, where $i=\sqrt{-1}$.

For $R e(s)>1$ the Euler product representation for Eq. (1) can be expressed in the form [2]:

$$
L(s, \chi)=\prod_{p}\left(1-\chi(p) p^{-s}\right)^{-1} .
$$

Let the primitive character $\chi^{*}$ with the modulus $q>1$ such that [3]

$$
\chi(n)=\left\{\begin{array}{l}
\chi^{*}(n) \text { if } \operatorname{gcd}(n, q)=1, \\
0 \text { if } \operatorname{gcd}(n, q) \neq 1
\end{array}\right.
$$

By Eq. (3) and $\operatorname{Re}(s)>1$, we rewrite Eq. (1) as [3]:

$$
L(s, \chi)=L\left(s, \chi^{*}\right) \prod_{p \mid q}\left(1-\frac{\chi^{*}(p)}{p^{s}}\right),
$$

where

$$
L\left(s, \chi^{*}\right)=\prod_{p}\left(1-\chi^{*}(p) p^{-s}\right)^{-1} .
$$

Let $\chi_{0}$ be the principal character with the modulus $q>1$ such that $[2,3]$

$$
L\left(s, \chi_{0}\right)=\zeta(s) \prod_{p \mid q}\left(1-\frac{1}{p^{s}}\right)(\operatorname{Re}(s)>1),
$$

where the Riemann zeta function is denoted by [4]

$$
\zeta(s)=\sum_{n=1}^{\infty} s^{-n}(\operatorname{Re}(s)>1) .
$$


Let $\chi^{*}$ be a primitive character modulo $q$ with $q>1$. Then the product for $L(s, \chi)$ can be represented as follows [3]:

$$
L\left(s, \chi^{*}\right)=\left(\frac{q}{\pi}\right)^{-\frac{s+\hbar\left(\chi^{*}\right)}{2}} \cdot \frac{\xi\left(s, \chi^{*}\right)}{\Gamma\left(\frac{s+\hbar\left(\chi^{*}\right)}{2}\right)}
$$

subject to

$$
\hbar\left(\chi^{*}\right)=\left\{\begin{array}{l}
0 \text { if } \chi(-1)=1 \\
1 \text { if } \chi(-1)=-1
\end{array}\right.
$$

and

$$
\xi\left(s, \chi^{*}\right)=\xi\left(0, \chi^{*}\right) \cdot e^{B\left(\chi^{*}\right) s} \cdot \prod_{k=1}^{\infty}\left(1-\frac{s}{\rho_{k}}\right) e^{s / \rho_{k}}
$$

where $s \in \mathbb{C}, \xi\left(0, \chi^{*}\right) \neq 0, B\left(\chi^{*}\right)=\xi^{(1)} / \xi\left(0, \chi^{*}\right)$, $\Gamma$ is the gamma function [5], and $\rho_{k}$ run all zeros of $\xi\left(s, \chi^{*}\right)$.

As the above results, we may present the followings [3]:

Cases 1. For $q=1$,

$$
L(s, \chi)=\zeta(s) \quad(\operatorname{Re}(s)>1)
$$

Cases 2. For $\chi=\chi_{0}$ with the modulus $q>1$,

$$
L(s, \chi)=L\left(s, \chi_{0}\right)=\zeta(s) \prod_{p \mid q}\left(1-\frac{1}{p^{s}}\right)(R e(s)>1)
$$

Cases 3. Let $\chi^{*}$ be a primitive character modulo $q$ with $q>1$. Then

$$
L(s, \chi)=L\left(s, \chi^{*}\right)=\xi\left(0, \chi^{*}\right) \cdot\left(\frac{q}{\pi}\right)^{-\frac{s+\hbar\left(\chi^{*}\right)}{2}} \cdot e^{B\left(\chi^{*}\right) s} \cdot \frac{\prod_{k=1}^{\infty}\left(1-\frac{s}{\rho_{k}}\right) e^{s / \rho_{k}}}{\Gamma\left(\frac{s+\hbar\left(\chi^{*}\right)}{2}\right)} .
$$

For Cases 1 it is well known that Eq. (11) has a pole at $s=1$ with residue1 [6], the trivial zeros $s=-2 h$ with $h \in \mathbb{N}$ [7], and the nontrivial zeros $s_{k}=1 / 2+i \varpi_{k}$ for $k \in \mathbb{N}$ (proved proved by author in the different methods [8,9]), where $\varpi_{k}$ are the Riemann-Siegel zeros, confirmed by the Riemann-Siegel formula [10]. Let $\mathbb{Z}$ be the set of the integral numbers. For Cases 2 it is seen that Eq. (12) has the zeros $s=2 \pi i j /(\log p)$ with $p \mid q$ and $j \in \mathbb{Z}$ (see [11], p.460) and a pole at $s=1$ with residues $v(q) / q$ (see [2], p.334), where $v(q)$ is the Euler's totient function. For Cases 3 it is shown that Eq. (13) has simple zeros $s=-\hbar\left(\chi^{*}\right)-2 h$ with $h \in \mathbb{N}$ (see [11], p.460; [2] p.333) and the nontrivial zeros $\rho_{k}=\alpha_{k}+i \beta_{k}$, where $\beta_{k}$ are the 
Siegel zeros, confirmed by the Siegel formula [12]. Let $N(T, \chi)$ denote the number of zeros of Eq. (13) for $\operatorname{Re}(s)=1 / 2, T>2$ and $|\operatorname{Im}(s)| \leq T$ such that [13]

$$
N(T, \chi)=\frac{T}{\pi} \log \frac{q T}{2 \pi}-\frac{T}{\pi}+O\left(\frac{\log (q T)}{\log \log (q T)}\right) .
$$

It is clearly seen that the nontrivial zeros of Eq. (13) exist and that Eq. (13) has infinitely many nontrivial zeros by using Eq. (14).

Conjecture 1. The generalized Riemann conjecture for Eq. (1) states the real part of the nontrivial zeros is $1 / 2$.

According to Davenport (see [2], p.124), Conjecture 1 was formulated in 1884 by Adolf Piltz. There have been applicable to consider the Goldbach's conjecture by Hardy and Littlewood in 1923 [14, 15] and by Wang in 1962 [16]. Hardy and Littlewood [17, 18, 19] proved that if Conjecture 1 is true, almost all even numbers are sums of two primes and that every large odd number is the sum of three primes.

Recently, author have proposed two classes of the Riemann zeta function, which can be represented by (the first term was by Hadamard [9, 11, 20] and the second term was by author [9])

$$
\begin{aligned}
& \zeta(s) \\
& =\xi(s) / \Re(s) \\
& =\frac{\xi(0) e^{s \Im}}{(s-1) \Gamma\left(\frac{s}{2}+1\right)} \prod_{k=1}^{\infty}\left(1-\frac{s}{s_{k}}\right) e^{s / s_{k}} \\
& =\frac{\xi(1 / 2) e^{s \Im}}{(s-1) \Gamma\left(\frac{s}{2}+1\right)} \prod_{k=1}^{\infty}\left(1-\frac{s-\frac{1}{2}}{s_{k}-\frac{1}{2}}\right) e^{s / s_{k}} \\
& =\frac{\xi(s) e^{s \Im}}{(s-1) \Gamma\left(\frac{s}{2}+1\right)} \prod_{k=1}^{\infty} e^{s / s_{k}}
\end{aligned}
$$

provided that there exists the entire Riemann zeta function, given as (the first term was by Hadamard [9, 11, 20] and the second term was by author [9])

$$
\xi(s)=\xi(0) e^{s \Im_{0}} \prod_{k=1}^{\infty}\left(1-\frac{s}{s_{k}}\right) e^{s / s_{k}}=\xi(1 / 2) e^{s \Im_{0}} \prod_{k=1}^{\infty}\left(1-\frac{s-\frac{1}{2}}{s_{k}-\frac{1}{2}}\right) e^{s / s_{k}},
$$

where

$$
\begin{gathered}
\Re(s)=(s-1) \pi^{-s / 2} \Gamma(s / 2+1), \\
\Im=\log 2 \pi-1-\frac{\widetilde{a}}{2},
\end{gathered}
$$

and

$$
\Im_{0}=\log 2 \pi-1-\frac{\widetilde{a}}{2}-\frac{1}{2} \log \pi
$$


with the Euler's constant $\widetilde{a}$.

As is well known, Conjecture 1 is an unsolved important mathematical problem in analytic number theory up to now. By inspired by the above results to structure the variant of the product for the Riemann zeta function, the main targets of the paper are to propose a variant of the product for Eq. (13), to prove Conjecture 1 for Eq. (1) and to present a detailed account of applications of the Hardy-Littlewood and Wang theorems to the Goldbach's conjecture directly removing the need for Conjecture 1. The structure of the paper is given as follows. In Section 2, we introduce the results related to the Riemann zeta function and Dirichlet $L$-functions. In Section 3 , we give the detailed proof of Conjecture 1. In Section 4 we apply to obtain the properties via Dirichlet $L$-functions, get the representations for Wang theorems, and apply the Hardy-Littlewood theorems to obtain the Goldbach's conjecture. Finally, we draw the conclusion in Section 5.

\section{Fundamental RESUlts}

We now consider the variants of the products for the Riemann zeta function and Dirichlet $L$-functions.

2.1. A variant of the product for the Riemann zeta function. We now consider the variant of the product for the Riemann zeta function.

Lemma 1. There exists

$$
\begin{gathered}
\zeta(1) \neq 0, \\
\zeta(0)=-\frac{1}{2} \neq 0,
\end{gathered}
$$

and

$$
\zeta\left(\frac{1}{2}\right) \neq 0
$$

Proof. See the results of Landau [11].

Remark. Eq. (20) was proved in 1896 by Hadamard [21] and Vallee-Poussin [22]. Eqs. (21) and (22) were discussed in Landau [11].

It is proved by Riemann [4] that

$$
\zeta(s)=\frac{\xi(s)}{\Re(s)},
$$

where $[11,20]$

$$
\zeta(s)=\xi(s) / \Re(s)=\frac{\xi(0) e^{s \Im}}{(s-1) \Gamma\left(\frac{s}{2}+1\right)} \prod_{k=1}^{\infty}\left(1-\frac{s}{s_{k}}\right) e^{s / s_{k}}
$$


and $[8,9]$

$$
\zeta(s)=\xi(s) / \Re(s)=\frac{\xi(1 / 2) e^{s \Im}}{(s-1) \Gamma\left(\frac{s}{2}+1\right)} \prod_{k=1}^{\infty}\left(1-\frac{s-\frac{1}{2}}{s_{k}-\frac{1}{2}}\right) e^{s / s_{k}}
$$

where

$$
\Re(s)=(s-1) \pi^{-s / 2} \Gamma(s / 2+1),
$$

and

$$
\Im=\log 2 \pi-1-\widetilde{a} / 2
$$

with the Euler's constant $\widetilde{a}$.

Lemma 2. There exists

$$
\xi(1)=\xi(0)=\frac{1}{2} \neq 0
$$

and

$$
\xi\left(\frac{1}{2}\right) \neq 0
$$

Proof. See the results of Broughan (see [23], p.49).

Lemma 3. Let $\Im_{0}=\log 2 \pi-1-\frac{\widetilde{a}}{2}-\frac{1}{2} \log \pi$, where $\widetilde{a}$ is the Euler's constant. Then two classes of the entire Riemann zeta function are equivalent:

$$
\xi(s)=\xi(0) e^{s \Im_{0}} \prod_{k=1}^{\infty}\left(1-\frac{s}{s_{k}}\right) e^{s / s_{k}}
$$

and

$$
\xi(s)=\xi(1 / 2) e^{s \Im_{0}} \prod_{k=1}^{\infty}\left(1-\frac{s-\frac{1}{2}}{s_{k}-\frac{1}{2}}\right) e^{s / s_{k}},
$$

where $s_{k}$ run the zeros for $\xi(s), s \in \mathbb{C}$ and $k \in \mathbb{N}$. 
Proof. According to Hadamard [20] and Landau [11], we have

$$
\begin{aligned}
& \xi(s) \\
& =\xi(0) e^{s \Im_{0}} \prod_{k=1}^{\infty}\left(1-\frac{s}{s_{k}}\right) e^{s / s_{k}} \\
& =\xi(0) e^{s \Im_{0}} \prod_{k=1}^{\infty} \frac{s_{k}-s}{s_{k}} e^{s / s_{k}} \\
& =\xi(0) e^{s \Im_{0}} \prod_{k=1}^{\infty}\left(\frac{s_{k}-\frac{1}{2}}{s_{k}-\frac{1}{2}} \cdot \frac{s_{k}-s}{s_{k}}\right) e^{s / s_{k}} \\
& =\xi(0) e^{s \Im_{0}} \prod_{k=1}^{\infty} \frac{s_{k}-\frac{1}{2}}{s_{k}} \prod_{k=1}^{\infty}\left(\frac{s_{k}-s}{s_{k}-\frac{1}{2}}\right) e^{s / s_{k}} \\
& =\xi(0) e^{s \Im_{0}} \prod_{k=1}^{\infty} \frac{s_{k}-\frac{1}{2}}{s_{k}} \prod_{k=1}^{\infty}\left(1-\frac{s-\frac{1}{2}}{s_{k}-\frac{1}{2}}\right) e^{s / s_{k}} \\
& =\xi(0) e^{s \Im_{0}} \prod_{k=1}^{\infty}\left(1-\frac{1}{2 s_{k}}\right) \prod_{k=1}^{\infty}\left(1-\frac{s-\frac{1}{2}}{s_{k}-\frac{1}{2}}\right) e^{s / s_{k}} \\
& =\xi(0) e^{\left(s-\frac{1}{2}\right) \Im_{0}} \prod_{k=1}^{\infty}\left(1-\frac{s-\frac{1}{2}}{s_{k}-\frac{1}{2}}\right) e^{s / s_{k}}
\end{aligned}
$$

where

$$
\xi\left(\frac{1}{2}\right)=\xi(0) e^{\frac{\Im_{0}}{2}} \prod_{k=1}^{\infty}\left(1-\frac{1}{2 s_{k}}\right) e^{1 / 2 s_{k}}=\xi(0) \prod_{k=1}^{\infty}\left(1-\frac{1}{2 s_{k}}\right),
$$

which is derived by $[6,11,20]$

$$
\xi(s)=\xi(0) e^{s \Im_{0}} \prod_{k=1}^{\infty}\left(1-\frac{s}{s_{k}}\right) e^{s / s_{k}}=\xi(0) \prod_{k=1}^{\infty}\left(1-\frac{s}{s_{k}}\right)
$$

and

$$
e^{\frac{\Im_{0}}{2}} \prod_{k=1}^{\infty} e^{1 / 2 s_{k}}=1
$$

Thus, the proof of Lemma 3 is completed.

Lemma 4. Assume that $s \in \mathbb{C}, k \in \mathbb{N}$, and $\Im_{0}=\log 2 \pi-1-\widetilde{a} / 2$, where $\widetilde{a}$ is the Euler's constant. Then two classes of the Riemann zeta function are equivalent:

$$
\zeta(s)=\frac{\xi(0) e^{s \Im}}{(s-1) \Gamma\left(\frac{s}{2}+1\right)} \prod_{k=1}^{\infty}\left(1-\frac{s}{s_{k}}\right) e^{s / s_{k}}
$$

and $[5,9]$

$$
\zeta(s)=\frac{\xi(1 / 2) e^{s \Im}}{(s-1) \Gamma\left(\frac{s}{2}+1\right)} \prod_{k=1}^{\infty}\left(1-\frac{s-\frac{1}{2}}{s_{k}-\frac{1}{2}}\right) e^{s / s_{k}}
$$


Proof. By Eq. (23), Lemma 3 and $\Re(s)=(s-1) \pi^{-s / 2} \Gamma(s / 2+1)$, we obtain the required results.

Remark. For the detailed proofs of Lemmas 3 and 4, see [8, 9].

According to Patterson [23] we have

$$
s(s-1) \Gamma\left(\frac{s}{2}\right) \zeta(s)=\prod_{k=1}^{\infty}\left(1-\frac{s}{s_{k}^{*}}\right)\left(1-\frac{s}{1-s_{k}^{*}}\right)
$$

such that [8]

$$
\xi(s)=\frac{1}{2} \prod_{k=1}^{\infty}\left(1-\frac{s}{s_{k}^{*}}\right)\left(1-\frac{s}{1-s_{k}^{*}}\right)=\xi(0) \prod_{k=1}^{\infty}\left(1-\frac{s}{s_{k}^{*}}\right)\left(1-\frac{s}{1-s_{k}^{*}}\right),
$$

where $s \in \mathbb{C}, k \in \mathbb{N}$, and

$$
s_{k}^{*}=\operatorname{Re}\left(s_{k}\right)+i\left|\operatorname{Im}\left(s_{k}\right)\right| .
$$

It is proved by author [8] that for $s \in \mathbb{C}$,

$$
\xi(s)=\xi(0) \prod_{k=1}^{\infty}\left(1-\frac{s}{s_{k}^{*}}\right)\left(1-\frac{s}{1-s_{k}^{*}}\right)=\xi\left(\frac{1}{2}\right) \prod_{k=1}^{\infty}\left[1-\frac{\left(s-\frac{1}{2}\right)^{2}}{\left(s_{k}^{*}-\frac{1}{2}\right)^{2}}\right]
$$

which implies from Eq. (23) that [8]

$$
\begin{gathered}
\zeta(s)=\frac{\xi(s)}{\Re(s)}=\frac{\xi(0) \pi^{s / 2}}{(s-1) \Gamma(s / 2+1)} \prod_{k=1}^{\infty}\left(1-\frac{s}{s_{k}^{*}}\right)\left(1-\frac{s}{1-s_{k}^{*}}\right), \\
\zeta(s)=\frac{\xi(s)}{\Re(s)}=\frac{\xi(0) \pi^{s / 2}}{(s-1) \Gamma(s / 2+1)} \prod_{k=1}^{\infty}\left[1-\frac{s(1-s)}{s_{k}^{*}\left(1-s_{k}^{*}\right)}\right],
\end{gathered}
$$

and $[8]$

$$
\zeta(s)=\frac{\xi(s)}{\Re(s)}=\frac{\xi\left(\frac{1}{2}\right) \pi^{s / 2}}{(s-1) \Gamma(s / 2+1)} \prod_{k=1}^{\infty}\left[1-\frac{\left(s-\frac{1}{2}\right)^{2}}{\left(s_{k}^{*}-\frac{1}{2}\right)^{2}}\right],
$$

where $s \in \mathbb{C}, s \neq 1$, and

$$
\Re(s)=(s-1) \pi^{-s / 2} \Gamma(s / 2+1) .
$$

Now, from Eq. (42), we see that $s_{k}, 1-s_{k}, s_{k}^{*}$ and $1-s_{k}^{*}$ are the zeros for $\xi(s)$ and the nontrivial zeros for $\zeta(s)[8]$. Moreover, $\xi(s)$ is a meromorphic continuation to the entire complex plane $s$ with pole of residue 1 [6], and $\xi(s)$ is an integral function of order 1 and simple in the entire complex plane $s$ [8]. By Eqs. (31) and (44), it is proved that $\operatorname{Re}\left(s_{k}\right)=1 / 2$, in other words that the Riemann conjecture is true (for the detailed proof, see $[8,9])$. 
2.2. A variant of the product for the Dirichlet $L$-functions. In this part we present the variant of the product for the Dirichlet $L$-functions. We now denote the primitive character modulo $q$ with $q>1$ by $\chi^{*}=\chi^{*}(n)$.

Lemma 5. If $\chi^{*}$ is a primitive character modulo $q$ with $q>1$ and $\chi^{*}(n) \neq \chi_{0}$, then

$$
\begin{aligned}
& L\left(1, \chi^{*}\right) \neq 0, \\
& L\left(0, \chi^{*}\right) \neq 0
\end{aligned}
$$

and

$$
L\left(\frac{1}{2}, \chi^{*}\right) \neq 0
$$

Proof. For the detailed proof of Eq. (46), see the work of Dirichlet [1,3]. Moreover, Eqs. (47) and (48) were proved in [25].

Lemma 6. If $\chi^{*}$ is a primitive character modulo $q$ with $q>1$, then we have that

$$
\xi\left(s, \chi^{*}\right)=L\left(s, \chi^{*}\right) \cdot\left(\frac{q}{\pi}\right)^{\frac{s+\hbar\left(\chi^{*}\right)}{2}} \cdot \Gamma\left(\frac{s+\hbar\left(\chi^{*}\right)}{2}\right),
$$

is an entire function of order 1 , where $s \in \mathbb{C}$ and $\hbar\left(\chi^{*}\right)$ is defined in (9).

Proof. For the detailed proof of Lemma 6, see [3].

Lemma 7. If $\chi^{*}$ is a primitive character modulo $q$ with $q>1$, then we have

$$
\begin{aligned}
& \xi\left(1, \chi^{*}\right) \neq 0, \\
& \xi\left(0, \chi^{*}\right) \neq 0
\end{aligned}
$$

and

$$
\xi\left(\frac{1}{2}, \chi^{*}\right) \neq 0
$$

Proof. For the detailed proofs of Eqs. (50), (51) and (52), see [11].

Lemma 8. Suppose that $\chi^{*}$ is a primitive character modulo $q$ with $q>1$ and $\rho_{k}$ run the nontrivial zeros of $L\left(s, \chi^{*}\right)$. Then there is

$$
\xi\left(s, \chi^{*}\right)=\xi\left(0, \chi^{*}\right) e^{B\left(\chi^{*}\right) s} \prod_{k=1}^{\infty}\left(1-\frac{s}{\rho_{k}}\right) e^{s / \rho_{k}},
$$

where $s \in \mathbb{C}$ and

$$
B\left(\chi^{*}\right)=\frac{\xi^{(1)}}{\xi}\left(0, \chi^{*}\right)
$$


Proof. For the detailed proof of Lemma 8, see [3].

Lemma 9. Suppose that $\chi^{*}$ is a primitive character modulo $q$ with $q>1$ and $\rho_{k}$ run the nontrivial zeros of $L\left(s, \chi^{*}\right)$. Then there is

$$
\frac{\xi^{(1)}}{\xi}\left(s, \chi^{*}\right)=B\left(\chi^{*}\right)+\sum_{k=1}^{\infty}\left(\frac{1}{s-\rho_{k}}+\frac{1}{\rho_{k}}\right) .
$$

Proof. For the detailed proof of Lemma 9, see [3].

Lemma 10. Suppose that $\chi^{*}$ is a primitive character modulo $q$ with $q>1$ and $\rho_{k}$ run the nontrivial zeros of $L\left(s, \chi^{*}\right)$. Then we have for $s \in \mathbb{C}$,

$$
L\left(s, \chi^{*}\right)=B\left(\chi^{*}\right) \Phi\left(s, \hbar\left(\chi^{*}\right)\right) L\left(1-s, \overline{\chi^{*}}\right),
$$

and

$$
\xi\left(s, \chi^{*}\right)=\varepsilon\left(\chi^{*}\right) \xi\left(1-s, \overline{\chi^{*}}\right)
$$

provided that

$$
\Phi\left(s, \hbar\left(\chi^{*}\right)\right)=2^{s} \pi^{1 / 2-s} \Gamma(1-s) \sin \frac{\pi}{2}\left(s+\hbar\left(\chi^{*}\right)\right),
$$

where $\hbar\left(\chi^{*}\right)$ is defined in (9),

$$
\varepsilon\left(\chi^{*}\right)=\frac{\tau\left(\chi^{*}\right)}{i^{\hbar\left(\chi^{*}\right)} \sqrt{q}},
$$

the Gauss sum $\tau\left(\chi^{*}\right)$ of $\chi^{*}$ is denoted by

$$
\tau\left(\chi^{*}\right)=\sum_{\theta=1}^{q} \chi^{*}(\theta) e^{\theta / q}
$$

and $\overline{\chi^{*}}$ is the complex conjugate character to $\chi^{*}$.

Proof. For the detailed proof of Lemma 10, see [2, 3].

Lemma 11. Suppose that $\chi^{*}$ is a primitive character modulo $q$ with $q>1$. Then we have

$$
\left|\tau\left(\chi^{*}\right)\right|=\sqrt{q}
$$

Proof. For the detailed proof of Lemma 11, see [3].

Thus, we see that

$$
\tau\left(\chi^{*}\right) \neq 0
$$

if $\chi^{*}$ is a primitive character modulo $q$ with $q>1$. 
Theorem 1. Suppose that $\chi^{*}$ is a primitive character modulo $q$ with $q>1$ and $\rho_{k}$ run the nontrivial zeros of $L\left(s, \chi^{*}\right)$ with $k \in \mathbb{N}$. Then there exist the equivalent representations:

$$
\xi\left(s, \chi^{*}\right)=\xi\left(0, \chi^{*}\right) e^{B\left(\chi^{*}\right) s} \prod_{k=1}^{\infty}\left(1-\frac{s}{\rho_{k}}\right) e^{s / \rho_{k}},
$$

and

$$
\xi\left(s, \chi^{*}\right)=\xi\left(\frac{1}{2}, \chi^{*}\right) e^{\left(s-\frac{1}{2}\right) B\left(\chi^{*}\right)} \prod_{k=1}^{\infty}\left(1-\frac{s-\frac{1}{2}}{\rho_{k}-\frac{1}{2}}\right) e^{(s-1 / 2) / \rho_{k}},
$$

where $s \in \mathbb{C}$ and

$$
B\left(\chi^{*}\right)=\frac{\xi^{(1)}}{\xi}\left(0, \chi^{*}\right) .
$$

Proof. From Eqs. (51) and (52),

$$
\xi\left(\frac{1}{2}, \chi^{*}\right)=\xi\left(0, \chi^{*}\right) e^{\frac{B\left(\chi^{*}\right)}{2}} \prod_{k=1}^{\infty}\left(1-\frac{1}{2 \rho_{k}}\right) \prod_{k=1}^{\infty} e^{1 /\left(2 \rho_{k}\right)} \neq 0 .
$$

By Lemma 8, we get

$$
\begin{aligned}
& \xi\left(s, \chi^{*}\right) \\
& =\xi\left(0, \chi^{*}\right) e^{B\left(\chi^{*}\right) s} \prod_{k=1}^{\infty} e^{s / \rho_{k}} \prod_{k=1}^{\infty}\left(1-\frac{s}{\rho_{k}}\right) \\
& =\xi\left(0, \chi^{*}\right) e^{B\left(\chi^{*}\right) s} \prod_{k=1}^{\infty} e^{s / \rho_{k}} \prod_{k=1}^{\infty}\left(\frac{\rho_{k}-s}{\rho_{k}}\right) \\
& =\xi\left(0, \chi^{*}\right) e^{B\left(\chi^{*}\right) s} \prod_{k=1}^{\infty} e^{s / \rho_{k}} \prod_{k=1}^{\infty}\left(\frac{\rho_{k}-\frac{1}{2}}{\rho_{k}-\frac{1}{2}} \cdot \frac{\rho_{k}-s}{\rho_{k}}\right) \\
& =\xi\left(0, \chi^{*}\right) e^{B\left(\chi^{*}\right) s} \prod_{k=1}^{\infty} e^{s / \rho_{k}} \prod_{k=1}^{\infty}\left(\frac{\rho_{k}-\frac{1}{2}}{\rho_{k}} \cdot \frac{\rho_{k}-s}{\rho_{k}-\frac{1}{2}}\right) \\
& =\xi\left(0, \chi^{*}\right) e^{B\left(\chi^{*}\right) s} \prod_{k=1}^{\infty} \frac{\rho_{k}-\frac{1}{2}}{\rho_{k}} \prod_{k=1}^{\infty} e^{s / \rho_{k}} \prod_{k=1}^{\infty}\left(\frac{\rho_{k}-s}{\rho_{k}-\frac{1}{2}}\right) \\
& =\xi\left(0, \chi^{*}\right) e^{B\left(\chi^{*}\right) s} \prod_{k=1}^{\infty} \frac{\rho_{k}-\frac{1}{2}}{\rho_{k}} \prod_{k=1}^{\infty} e^{s / \rho_{k}} \prod_{k=1}^{\infty}\left[\frac{\left(\rho_{k}-\frac{1}{2}\right)-\left(s-\frac{1}{2}\right)}{\rho_{k}-\frac{1}{2}}\right] \\
& =\xi\left(0, \chi^{*}\right) e^{B\left(\chi^{*}\right) s} \prod_{k=1}^{\infty} \frac{\rho_{k}-\frac{1}{2}}{\rho_{k}} \prod_{k=1}^{\infty} e^{s / \rho_{k}} \prod_{k=1}^{\infty}\left(1-\frac{s-\frac{1}{2}}{\rho_{k}-\frac{1}{2}}\right) \\
& =\xi\left(0, \chi^{*}\right) e^{B\left(\chi^{*}\right) s} \prod_{k=1}^{\infty} \frac{\rho_{k}-\frac{1}{2}}{\rho_{k}} \prod_{k=1}^{\infty}\left(1-\frac{s-\frac{1}{2}}{\rho_{k}-\frac{1}{2}}\right) e^{s / \rho_{k}} \\
& =\xi\left(0, \chi^{*}\right) e^{s B\left(\chi^{*}\right)} \prod_{k=1}^{\infty}\left(1-\frac{1}{2 \rho_{k}}\right) \prod_{k=1}^{\infty}\left(1-\frac{s-\frac{1}{2}}{\rho_{k}-\frac{1}{2}}\right) e^{s / \rho_{k}} \\
& =\xi\left(\frac{1}{2}, \chi^{*}\right) e^{\left(s-\frac{1}{2}\right) B\left(\chi^{*}\right)} \prod_{k=1}^{\infty}\left(1-\frac{s-\frac{1}{2}}{\rho_{k}-\frac{1}{2}}\right) e^{(s-1 / 2) / \rho_{k}},
\end{aligned}
$$


which is the required result.

Theorem 2. Suppose that $\chi^{*}$ is a primitive character modulo $q$ with $q>1$ and $\rho_{k}$ run the nontrivial zeros of $L\left(s, \chi^{*}\right)$ with $k \in \mathbb{N}$. Then there exist the equivalent representations:

$$
L\left(s, \chi^{*}\right)=\frac{\xi\left(0, \chi^{*}\right) \cdot\left(\frac{q}{\pi}\right)^{-\frac{s+\hbar\left(\chi^{*}\right)}{2}} \cdot e^{B\left(\chi^{*}\right) s}}{\Gamma\left(\frac{s+\hbar\left(\chi^{*}\right)}{2}\right)} \cdot \prod_{k=1}^{\infty}\left(1-\frac{s}{\rho_{k}}\right) e^{s / \rho_{k}},
$$

and

$$
L\left(s, \chi^{*}\right)=\frac{\xi\left(\frac{1}{2}, \chi^{*}\right) \cdot\left(\frac{q}{\pi}\right)^{-\frac{s+\hbar\left(\chi^{*}\right)}{2}} \cdot e^{\left(s-\frac{1}{2}\right) B\left(\chi^{*}\right)}}{\Gamma\left(\frac{s+\hbar\left(\chi^{*}\right)}{2}\right)} \cdot \prod_{k=1}^{\infty}\left(1-\frac{s-\frac{1}{2}}{\rho_{k}-\frac{1}{2}}\right) e^{(s-1 / 2) / \rho_{k}},
$$

where $s \in \mathbb{C}$ and

$$
B\left(\chi^{*}\right)=\frac{\xi^{(1)}}{\xi}\left(0, \chi^{*}\right)
$$

Proof. With use of Lemma 6 and Theorem 1, we present [11]

$$
L\left(s, \chi^{*}\right)=\frac{\xi\left(0, \chi^{*}\right) \cdot\left(\frac{q}{\pi}\right)^{-\frac{s+\hbar\left(\chi^{*}\right)}{2}} \cdot e^{B\left(\chi^{*}\right) s}}{\Gamma\left(\frac{s+\hbar\left(\chi^{*}\right)}{2}\right)} \cdot \prod_{k=1}^{\infty}\left(1-\frac{s}{\rho_{k}}\right) e^{s / \rho_{k}},
$$

and

$$
L\left(s, \chi^{*}\right)=\frac{\xi\left(\frac{1}{2}, \chi^{*}\right) \cdot\left(\frac{q}{\pi}\right)^{-\frac{s+\hbar\left(\chi^{*}\right)}{2}} \cdot e^{\left(s-\frac{1}{2}\right) B\left(\chi^{*}\right)}}{\Gamma\left(\frac{s+\hbar\left(\chi^{*}\right)}{2}\right)} \cdot \prod_{k=1}^{\infty}\left(1-\frac{s-\frac{1}{2}}{\rho_{k}-\frac{1}{2}}\right) e^{(s-1 / 2) / \rho_{k}}
$$

which are the desired results, where $s \in \mathbb{C}$.

Remark. There exists (see [2], p.83)

$$
B\left(\chi^{*}\right)=\frac{\xi^{(1)}}{\xi}\left(0, \chi^{*}\right)=-\frac{\xi^{(1)}}{\xi}\left(1, \chi^{*}\right)=-B\left(\overline{\chi^{*}}\right)-\sum_{k=1}^{\infty}\left(\frac{1}{1-\rho_{k}}+\frac{1}{\rho_{k}}\right) .
$$

Lemma 12. Let $\chi^{*}$ be a primitive character modulo $q$ with $q>1 . L\left(s, \chi^{*}\right)$ has an infinity of zeros $\rho_{k}$ in the critical strip $0 \leq R e(s) \leq 1$.

Proof. See the result of Davenport (see [2], p.82). 
2.3. The Goldbach's problems. We now introduce the Hardy-Littlewood and Wang's theorems with the need of Conjecture 1.

Lemma 13. (Hardy-Littlewood Theorem I) [14] If Conjecture 1 is true, then every odd number $m>5$ is the sum of three primes.

Proof. See the work of Hardy and Littlewood [14] and the paper of Deshouillers and coauthors [15] under the condition of truth of Conjecture 1. Lemma 13 was proved in 2013 by Helfgott [26] without Conjecture 1 and a detailed account of the numerical verification for Lemma 13 was shown by Helfgott and Platt in 2013 [27].

For the sake of brevity, we denote the following proposition by $(1, \mathbb{X})[16,28]$ :

Every sufficiently large even integer is a sum of a prime and an almost prime of at most $\mathbb{X}$ prime divisors.

Lemma 14. (Wang Theorem I) [16]

If Conjecture 1 is true, then $(1,4)$ is valid, where $\delta_{2} \geq 3.237 / 2.237$.

Proof. See the work of Wang [16].

Lemma 15. (Wang Theorem II) [16]

If Conjecture 1 is true, then $(1,3)$ is valid, where $\delta_{1} \geq 2.475 / 1.475$.

Proof. See the work of Wang [16].

In fact, Chen proved in 1973 and 1978 that $(1,2)$ is true [28, 29]. The records of verification of the strong Goldbach's conjecture holds to $N_{1}=4 \times 10 \wedge 18$ [30].

Lemma 16. (Hardy-Littlewood Theorem II) [17]

If Conjecture 1 is true, every even number $m>2$ is sums of two primes.

Proof. See the work of Hardy and Littlewood [17]. Based on it, the work of Granville [18] gives the detailed proof of Lemma 16 on the condition of truth of Conjecture 1.

\section{A detailed proof For the Generalized Riemann conjecture}

We now apply the variant of the product for the Dirichlet $L$-functions to present the complete proof for Conjecture 1.

3.1. Family 1: $\chi=\chi_{0}$ and $q=1$. When $\chi=\chi_{0}$ and $q=1$, we have [3]

$$
\zeta(s)=\sum_{n=1}^{\infty} s^{-n}(\operatorname{Re}(s)>1) .
$$


According to Lemma 3, we have the representation

$$
\begin{aligned}
& \zeta(s) \\
& =\frac{\xi(0) e^{s \Im}}{(s-1) \Gamma\left(\frac{s}{2}+1\right)} \prod_{k=1}^{\infty}\left(1-\frac{s}{s_{k}}\right) e^{s / s_{k}} \\
& =\frac{\xi(1 / 2) e^{s \Im}}{(s-1) \Gamma\left(\frac{s}{2}+1\right)} \prod_{k=1}^{\infty}\left(1-\frac{s-\frac{1}{2}}{s_{k}-\frac{1}{2}}\right) e^{s / s_{k}} \\
& =\frac{\xi(s) e^{s \Im}}{(s-1) \Gamma\left(\frac{s}{2}+1\right)} \prod_{k=1}^{\infty} e^{s / s_{k}}
\end{aligned}
$$

where $s \in \mathbb{C}$ and $s \neq 1$, and so we have

$$
\xi(s)=\xi(0) \prod_{k=1}^{\infty}\left(1-\frac{s}{s_{k}}\right)=\xi(1 / 2) \prod_{k=1}^{\infty}\left(1-\frac{s-\frac{1}{2}}{s_{k}-\frac{1}{2}}\right)
$$

and

$$
\Xi(\beta)=\xi\left(\frac{1}{2}+i \beta\right)=\xi(0) \prod_{k=1}^{\infty}\left(1-\frac{\frac{1}{2}+i \beta}{s_{k}}\right)=\xi(1 / 2) \prod_{k=1}^{\infty}\left(1-\frac{i \beta}{s_{k}-\frac{1}{2}}\right),
$$

where $\xi(0) \neq 0$ and $\xi(1 / 2) \neq 0$ (for the details, see Lemma 2).

It is well knows that Eqs. (76) and (77) are the entire functions of order 1.

By Eqs. (75) and (76), we have

$$
\zeta(s)=0
$$

such that

$$
\begin{gathered}
s-1 \neq 0, \\
\xi(s)=0
\end{gathered}
$$

and

$$
\frac{1}{\Gamma\left(\frac{s}{2}+1\right)}=0
$$

With use of Eq. (77), we have [8, 9]

$$
\Xi\left(\varpi_{k}\right)=\xi(1 / 2) \prod_{k=1}^{\infty}\left(1-\frac{i \varpi_{k}}{s_{k}-\frac{1}{2}}\right)=0 .
$$

Since Eq. (76) is an entire function of order 1, it follows that

$$
1-\frac{i \varpi_{k}}{s_{k}-\frac{1}{2}}=0
$$

where $k \in \mathbb{N}$. 
From Eq. (83), we have

$$
s_{k}=1 / 2+i \varpi_{k}
$$

and $[8,9]$

$$
\widetilde{s_{k}}=\frac{1}{2} \pm i\left|\varpi_{k}\right|
$$

where $k \in \mathbb{N}, i=\sqrt{-1}$, and $\varpi_{k}$ are the Riemann-Siegel zeros.

Putting Eq. (76) into Eq. (84), we get

$$
\xi(s)=\xi(1 / 2) \prod_{k=1}^{\infty}\left(1-\frac{s-\frac{1}{2}}{i \varpi_{k}}\right) .
$$

Substituting $s_{k}=\omega_{k}+i \varpi_{k}$ into Eq. (87), we get

$$
\xi\left(\omega_{k}+i \varpi_{k}\right)=\xi(1 / 2) \prod_{k=1}^{\infty}\left(1-\frac{\omega_{k}+i \varpi_{k}-\frac{1}{2}}{i \varpi_{k}}\right)=0 .
$$

Since Eq. (77) is an entire function of order 1, it follows that

$$
1-\frac{\omega_{k}+i \varpi_{k}-\frac{1}{2}}{i \varpi_{k}}=0
$$

which leads to

$$
\omega_{k}=1 / 2,
$$

or, alternatively,

$$
s_{k}=1 / 2+i \varpi_{k}
$$

With use of Eq. (79), Eq. (75) has a pole at $s=1$ with residue 1 [6]. By Eq. (81), Eq. (75) has the trivial zeros $s=-2 h$ with $h \in \mathbb{N}[6]$. In view of Eq. (89), we find that Eq. (75) has the nontrivial zeros $s_{k}=1 / 2+i \varpi_{k}[8,9]$.

3.2. Family 2: $\chi=\chi_{0}$ and $q>1$. When $\chi=\chi_{0}$ and $q>1$, we get [3]

$$
L\left(s, \chi_{0}\right)=\zeta(s) \prod_{p \mid q}\left(1-\frac{1}{p^{s}}\right)(R e(s)>1) .
$$


By Lemmas 3, 4, (75) and (91), we show that

$$
\begin{aligned}
& L\left(s, \chi_{0}\right) \\
& =\zeta(s) \prod_{p \mid q}\left(1-\frac{1}{p^{s}}\right) \\
& =\frac{\xi(0) e^{s \Im}}{(s-1) \Gamma\left(\frac{s}{2}+1\right)} \prod_{k=1}^{\infty}\left(1-\frac{s}{s_{k}}\right) e^{s / s_{k}} \prod_{p \mid q}\left(1-\frac{1}{p^{s}}\right) \\
& =\frac{\xi(1 / 2) e^{s \Im}}{(s-1) \Gamma\left(\frac{s}{2}+1\right)} \prod_{k=1}^{\infty}\left(1-\frac{s-\frac{1}{2}}{s_{k}-\frac{1}{2}}\right) e^{s / s_{k}} \prod_{p \mid q}\left(1-\frac{1}{p^{s}}\right) \\
& =\frac{\xi(s) e^{s \Im}}{(s-1) \Gamma\left(\frac{s}{2}+1\right)} \prod_{p \mid q}\left(1-\frac{1}{p^{s}}\right) \prod_{k=1}^{\infty} e^{s / s_{k}},
\end{aligned}
$$

where $s \in \mathbb{C}$ and $s \neq 1$.

By Eq. (92) and Lemma 2, we have

$$
L\left(s, \chi_{0}\right)=0
$$

such that

$$
1-\frac{1}{p^{s}}=0
$$

and

$$
\zeta(s)=0 .
$$

By Family 1, it is clearly seen that Eq. (95) has the nontrivial zeros $s_{k}=1 / 2+i \varpi_{k}$ for $k \in \mathbb{N}[8,9]$ and the trivial zeros $s=-2 h$ with $h \in \mathbb{N}[6]$, and a pole at $s=1$ with residue $v(q) / q=\prod_{p \mid q}\left(1-\frac{1}{p}\right)([3]$, p.334), where $v(q)$ is the Euler's totient function. From Eq. (94) it is shown that Eq. (93) has the pure imaginary zeros $s=2 \pi i j /(\log p)$ with $p \mid q$ and $j \in \mathbb{Z}$ (see [11], p.460).

3.3. Family 3: $\chi=\chi^{*}$ and $\chi^{*}(-1)=1$. When $\chi \neq \chi_{0}$ and $\chi(-1)=1$, we write [3]

$$
\xi\left(s, \chi^{*}\right)=L\left(s, \chi^{*}\right) \Gamma\left(\frac{s+k\left(\chi^{*}\right)}{2}\right)\left(\frac{q}{\pi}\right)^{\frac{s+k\left(\chi^{*}\right)}{2}}
$$

as

$$
\xi\left(s, \chi^{*}\right)=L\left(s, \chi^{*}\right) \Gamma\left(\frac{s}{2}\right)\left(\frac{q}{\pi}\right)^{\frac{s}{2}}
$$

with

$$
\xi\left(s, \chi^{*}\right)=\varepsilon\left(\chi^{*}\right) \xi\left(1-s, \overline{\chi^{*}}\right)
$$

where $s \in \mathbb{C}$,

$$
k\left(\chi^{*}\right)=0,
$$




$$
\varepsilon\left(\chi^{*}\right)=\frac{\tau\left(\chi^{*}\right)}{\sqrt{q}},
$$

and the Gauss sum of $\chi^{*}$ by

$$
\tau\left(\chi^{*}\right)=\sum_{\theta=1}^{q} \chi^{*}(\theta) e^{\theta / q} .
$$

Since $\xi\left(s, \chi^{*}\right)$ is an integral function of order 1 , we have

$$
\xi\left(s, \chi^{*}\right)=\xi\left(0, \chi^{*}\right) e^{B\left(\chi^{*}\right) s} \prod_{k=1}^{\infty}\left(1-\frac{s}{\rho_{k}}\right) e^{s / \rho_{k}}
$$

such that (see Theorem 1)

$$
\begin{aligned}
& \xi\left(s, \chi^{*}\right) \\
& =\xi\left(0, \chi^{*}\right) e^{B\left(\chi^{*}\right) s} \prod_{k=1}^{\infty} e^{s / \rho_{k}} \prod_{k=1}^{\infty}\left(1-\frac{s}{\rho_{k}}\right) \\
& =\xi(0, \chi) e^{B(\chi) s} \prod_{k=1}^{\infty}\left(1-\frac{1}{2 \rho_{k}}\right) \prod_{k=1}^{\infty}\left(1-\frac{s-\frac{1}{2}}{\rho_{k}-\frac{1}{2}}\right) e^{s / \rho_{k}} .
\end{aligned}
$$

By Eq. (102), we have

$$
\xi\left(\frac{1}{2}, \chi^{*}\right)=\xi\left(0, \chi^{*}\right) e^{\frac{B\left(\chi^{*}\right)}{2}} \prod_{k=1}^{\infty}\left(1-\frac{1}{2 \rho_{k}}\right) \prod_{k=1}^{\infty} e^{1 /\left(2 \rho_{k}\right)}
$$

such that

$$
\frac{\xi\left(s, \chi^{*}\right)}{\xi\left(\frac{1}{2}, \chi^{*}\right)}=\frac{\xi\left(0, \chi^{*}\right) e^{B\left(\chi^{*}\right) s} \prod_{k=1}^{\infty}\left(1-\frac{1}{2 \rho_{k}}\right) \prod_{k=1}^{\infty}\left(1-\frac{s-\frac{1}{2}}{\rho_{k}-\frac{1}{2}}\right) e^{s / \rho_{k}}}{\xi\left(0, \chi^{*}\right) e^{\frac{B\left(\chi^{*}\right)}{2}} \prod_{k=1}^{\infty}\left(1-\frac{1}{2 \rho_{k}}\right) \prod_{k=1}^{\infty} e^{1 /\left(2 \rho_{k}\right)}},
$$

where (see Lemma 7 for the details)

$$
\xi\left(0, \chi^{*}\right) \neq 0
$$

and

$$
\xi\left(\frac{1}{2}, \chi^{*}\right) \neq 0
$$

By Theorem 1, we arrive at

$$
\xi\left(s, \chi^{*}\right)=\xi\left(\frac{1}{2}, \chi^{*}\right) e^{B\left(\chi^{*}\right)\left(s-\frac{1}{2}\right)} \prod_{k=1}^{\infty}\left(1-\frac{s-\frac{1}{2}}{\rho_{k}-\frac{1}{2}}\right) e^{(s-1 / 2) / \rho_{k}} .
$$


From (97), (108) and Theorem 2, we suggest that

$$
\begin{aligned}
& L\left(s, \chi^{*}\right) \\
& =\left(\frac{q}{\pi}\right)^{-\frac{s}{2}} \frac{\xi\left(s, \chi^{*}\right)}{\Gamma\left(\frac{s}{2}\right)} \\
& =\frac{\left(\frac{q}{\pi}\right)^{-\frac{s}{2}} \cdot \xi\left(\frac{1}{2}, \chi^{*}\right) \cdot e^{B\left(\chi^{*}\right)\left(s-\frac{1}{2}\right)}}{\Gamma\left(\frac{s}{2}\right)} \prod_{k=1}^{\infty}\left(1-\frac{s-\frac{1}{2}}{\rho_{k}-\frac{1}{2}}\right) e^{(s-1 / 2) / \rho_{k}} .
\end{aligned}
$$

Since Eq. (108) is an integral function of order 1, Eq. (109) is an integral function of order 1.

Substituting $s=1 / 2+i \beta$ into Eq. (108), we have [11]

$$
\Xi\left(\beta, \chi^{*}\right)=\xi\left(1 / 2+i \beta, \chi^{*}\right)=\xi\left(\frac{1}{2}, \chi^{*}\right) e^{i \beta B\left(\chi^{*}\right)} \prod_{k=1}^{\infty}\left(1-\frac{i \beta}{\rho_{k}-\frac{1}{2}}\right) e^{i \beta / \rho_{k}} .
$$

It is shown that Eq. (66) is an integral function of order 1.

Taking $\beta=\beta_{k}$, we have from Eq. (66) that

$$
\Xi\left(\beta_{k}, \chi^{*}\right)=\xi\left(\frac{1}{2}, \chi^{*}\right) e^{i \beta_{k} B\left(\chi^{*}\right)} \prod_{k=1}^{\infty}\left(1-\frac{i \beta_{k}}{\rho_{k}-\frac{1}{2}}\right) e^{i \beta_{k} / \rho_{k}}=0 .
$$

By Eq. (67) and Lemma \%, we may arrive at

$$
1-\frac{i \beta_{k}}{\rho_{k}-\frac{1}{2}}=0
$$

and by Eq. (112) we get

$$
\rho_{k}=\frac{1}{2}+i \beta_{k}
$$

By Eq. (113), Eqs. (108), (109) and (110) can be rewritten as

$$
\begin{gathered}
\xi\left(s, \chi^{*}\right)=\xi\left(\frac{1}{2}, \chi^{*}\right) e^{B\left(\chi^{*}\right)\left(s-\frac{1}{2}\right)} \prod_{k=1}^{\infty}\left(1-\frac{s-\frac{1}{2}}{i \beta_{k}}\right) e^{(s-1 / 2) /\left(1 / 2+i \beta_{k}\right)}, \\
L\left(s, \chi^{*}\right)=\frac{\left(\frac{q}{\pi}\right)^{-\frac{s}{2}} \xi\left(\frac{1}{2}, \chi^{*}\right) e^{B\left(\chi^{*}\right)\left(s-\frac{1}{2}\right)}}{\Gamma\left(\frac{s}{2}\right)} \prod_{k=1}^{\infty}\left(1-\frac{s-\frac{1}{2}}{i \beta_{k}}\right) e^{(s-1 / 2) /\left(1 / 2+i \beta_{k}\right)},
\end{gathered}
$$

and

$$
\Xi\left(\beta, \chi^{*}\right)=\xi\left(1 / 2+i \beta, \chi^{*}\right)=\xi\left(\frac{1}{2}, \chi^{*}\right) e^{i \beta B\left(\chi^{*}\right)} \prod_{k=1}^{\infty}\left(1-\frac{\beta}{\beta_{k}}\right) e^{i \beta /\left(1 / 2+i \beta_{k}\right)},
$$

where $s \in \mathbb{C}$. 
From Eq. (60) and Lemma 6, we observe that

$$
L\left(s, \chi^{*}\right)=0,
$$

which leads to

$$
\frac{1}{\Gamma\left(\frac{s}{2}\right)}=0
$$

and

$$
\xi\left(s, \chi^{*}\right)=\xi\left(\frac{1}{2}, \chi^{*}\right) e^{B\left(\chi^{*}\right)\left(s-\frac{1}{2}\right)} \prod_{k=1}^{\infty}\left(1-\frac{s-\frac{1}{2}}{i \beta_{k}}\right) e^{(s-1 / 2) /\left(1 / 2+i \beta_{k}\right)}=0 .
$$

Making use of Eq. (118), we find that [2, 3, 11]

$$
s=-2 k,
$$

which are the trivial zeros of Eq. (109), where $k \in \mathbb{N} \cup\{0\}$.

Inserting $\rho_{k}=\alpha_{k}+i \beta_{k}$ into Eq. (119) implies that

$$
\begin{aligned}
& \xi\left(\alpha_{k}+i \beta_{k}, \chi^{*}\right) \\
& =\xi\left(\frac{1}{2}, \chi^{*}\right) e^{B\left(\chi^{*}\right)\left(\alpha_{k}+i \beta_{k}-\frac{1}{2}\right)} \prod_{k=1}^{\infty}\left(1-\frac{\alpha_{k}+i \beta_{k}-\frac{1}{2}}{i \beta_{k}}\right) e^{\left(\alpha_{k}+i \beta_{k}-1 / 2\right) /\left(1 / 2+i \beta_{k}\right)}, \\
& =0 .
\end{aligned}
$$

With Eq. (121) and Lemma 6, we arrive at

$$
1-\frac{\alpha_{k}+i \beta_{k}-\frac{1}{2}}{i \beta_{k}}=0,
$$

and we get

$$
\alpha_{k}=\frac{1}{2} \text {. }
$$

Thus,

$$
\rho_{k}=1 / 2+i \beta_{k}
$$

or, alternatively,

$$
\operatorname{Re}\left(\rho_{k}\right)=1 / 2 \text {. }
$$


3.4. Family 4: $\chi=\chi^{*}$ and $\chi^{*}(-1)=-1$. When $\chi=\chi^{*}$ and $\chi^{*}(-1)=-1$, we write [3]

$$
\xi\left(s, \chi^{*}\right)=L\left(s, \chi^{*}\right) \Gamma\left(\frac{s+k\left(\chi^{*}\right)}{2}\right)\left(\frac{q}{\pi}\right)^{\frac{s+k\left(\chi^{*}\right)}{2}}
$$

as

$$
\xi\left(s, \chi^{*}\right)=L\left(s, \chi^{*}\right) \Gamma\left(\frac{s+1}{2}\right)\left(\frac{q}{\pi}\right)^{\frac{s+1}{2}}
$$

with

$$
\xi\left(s, \chi^{*}\right)=\varepsilon\left(\chi^{*}\right) \xi\left(1-s, \overline{\chi^{*}}\right)
$$

where $s \in \mathbb{C}$,

$$
\begin{gathered}
k\left(\chi^{*}\right)=1 \\
\varepsilon\left(\chi^{*}\right)=\frac{\tau\left(\chi^{*}\right)}{\sqrt{q}},
\end{gathered}
$$

and the Gauss sum of $\chi^{*}$ by [3]

$$
\tau\left(\chi^{*}\right)=\sum_{\theta=1}^{q} \chi^{*}(\theta) e^{\theta / q}
$$

Since $\xi\left(s, \chi^{*}\right)$ is an integral function of order 1 , by Theorem 1, we have from Eqs. (102) and (108) that

$$
\begin{aligned}
& \xi\left(s, \chi^{*}\right) \\
& =\xi\left(0, \chi^{*}\right) e^{B\left(\chi^{*}\right) s} \prod_{k=1}^{\infty}\left(1-\frac{s}{\rho_{k}}\right) e^{s / \rho_{k}} \\
& =\xi\left(\frac{1}{2}, \chi^{*}\right) e^{B\left(\chi^{*}\right)\left(s-\frac{1}{2}\right)} \prod_{k=1}^{\infty}\left(1-\frac{s-\frac{1}{2}}{\rho_{k}-\frac{1}{2}}\right) e^{(s-1 / 2) / \rho_{k}} .
\end{aligned}
$$

Since Eq. (132) is an integral function of order $1, L\left(s, \chi^{*}\right)$ is an integral function of order 1.

Taking $s=1 / 2+i \beta$ into Eq. (132), we get

$$
\begin{aligned}
& \Xi\left(\beta, \chi^{*}\right) \\
& =\xi\left(1 / 2+i \beta, \chi^{*}\right) \\
& =\xi\left(0, \chi^{*}\right) e^{B\left(\chi^{*}\right) s} \prod_{k=1}^{\infty}\left(1-\frac{\frac{1}{2}+i \beta}{\rho_{k}}\right) e^{s / \rho_{k}} \\
& =\xi\left(\frac{1}{2}, \chi^{*}\right) e^{i \beta B\left(\chi^{*}\right)} \prod_{k=1}^{\infty}\left(1-\frac{i \beta}{\rho_{k}-\frac{1}{2}}\right) e^{i \beta / \rho_{k}} .
\end{aligned}
$$

We may find that Eq. (133) is an integral function of order 1. 
Substituting $\beta=\beta_{k}$ into Eq. (133), we have

$$
\Xi\left(\beta_{k}, \chi^{*}\right)=\xi\left(\frac{1}{2}, \chi^{*}\right) e^{i \beta_{k} B\left(\chi^{*}\right)} \prod_{k=1}^{\infty}\left(1-\frac{i \beta_{k}}{\rho_{k}-\frac{1}{2}}\right) e^{i \beta_{k} / \rho_{k}}=0 .
$$

By the virtue of Eq. (134) and Lemma 6, we give

$$
1-\frac{i \beta_{k}}{\rho_{k}-\frac{1}{2}}=0
$$

and by Eq. (135), we arrive at

$$
\rho_{k}=\frac{1}{2}+i \beta_{k}
$$

Combining Eqs. (127) and (132) and (136) and using Theorem 2, we have

$$
\begin{aligned}
& L\left(s, \chi^{*}\right) \\
& =\left(\frac{q}{\pi}\right)^{-\frac{s+1}{2}} \frac{\xi\left(s, \chi^{*}\right)}{\Gamma\left(\frac{s+1}{2}\right)} \\
& =\frac{\xi\left(\frac{1}{2}, \chi^{*}\right) \cdot\left(\frac{q}{\pi}\right)^{-\frac{s+1}{2}} \cdot e^{B\left(\chi^{*}\right)\left(s-\frac{1}{2}\right)}}{\Gamma\left(\frac{s+1}{2}\right)} \prod_{k=1}^{\infty}\left(1-\frac{s-\frac{1}{2}}{i \beta_{k}}\right) e^{(s-1 / 2) /\left(1 / 2+i \beta_{k}\right)} .
\end{aligned}
$$

In view of Eq. (137), we obtain

$$
\frac{1}{\Gamma\left(\frac{s+1}{2}\right)}=0
$$

and

$$
\xi\left(s, \chi^{*}\right)=\xi\left(\frac{1}{2}, \chi^{*}\right) \cdot e^{B\left(\chi^{*}\right)\left(s-\frac{1}{2}\right)} \prod_{k=1}^{\infty}\left(1-\frac{s-\frac{1}{2}}{i \beta_{k}}\right) e^{(s-1 / 2) /\left(1 / 2+i \beta_{k}\right)}=0 .
$$

By Eq. (138), we present [2, 3, 11]

$$
s=-2 k-1
$$

which is the trivial zeros of Eq. (137), where $k \in \mathbb{N} \cup\{0\}$.

Substituting $\rho_{k}=\alpha_{k}+i \beta_{k}$ into Eq. (139) we obtain

$$
\begin{aligned}
& \xi\left(\alpha_{k}+i \beta_{k}, \chi^{*}\right) \\
& =\xi\left(\frac{1}{2}, \chi^{*}\right) \cdot e^{B\left(\chi^{*}\right)\left(\alpha_{k}+i \beta_{k}-\frac{1}{2}\right)} \prod_{k=1}^{\infty}\left(1-\frac{\alpha_{k}+i \beta_{k}-\frac{1}{2}}{i \beta_{k}}\right) e^{\left(\alpha_{k}+i \beta_{k}-1 / 2\right) /\left(1 / 2+i \beta_{k}\right)} \\
& =0
\end{aligned}
$$

which implies, by Lemma 6, that

$$
1-\frac{\alpha_{k}+i \beta_{k}-\frac{1}{2}}{i \beta_{k}}=0
$$


Thus, by Eq. (123) we have

$$
\alpha_{k}=1 / 2
$$

such that

$$
\rho_{k}=1 / 2+i \beta_{k}
$$

By Lemma 12 and using the above results, we clearly see that the real part of the zeros in the critical trip is $1 / 2$.

It is shown that Conjecture 1 is true.

Hence, we finish the proof of Conjecture 1.

Remark. In short, we easily see the followings:

- When $\chi=\chi_{0}$ and $q=1, L(s, \chi)=\zeta(s)$ is extended to be a meromorphic continuation to the entire complex plane $s$, and has a pole at $s=1$ with residue 1 , the trivial zeros $s=-2 h$ with $h \in \mathbb{N}$, and the nontrivial zeros $s_{k}=1 / 2+i \varpi_{k}$ with $k \in \mathbb{N}$, which lie on the critical line $s=1 / 2$ and in the critical trip $0<\operatorname{Re}(s)<1$.

As shown in Section 1, Case 1 has been proved by authors in [8, 9].

- When $\chi=\chi_{0}$ and $q>1, L(s, \chi)=L\left(s, \chi_{0}\right)$ is extended to be a meromorphic continuation to the entire complex plane $s$, and has a pole at $s=1$ with residue $v(q) / q=\prod_{p \mid q}\left(1-p^{-1}\right)$, the pure imaginary zeros $s=2 \pi i j /(\log p)$ with $p \mid q$ and $j \in \mathbb{Z}$, the trivial zeros $s=-2 h$ with $h \in \mathbb{N}$, and the nontrivial zeros $s_{k}=1 / 2+i \varpi_{k}$ for $k \in \mathbb{N}$.

It is seen that Cases $\mathbf{2}$ and $\mathbf{1}$ have the same as the nontrivial zeros $s_{k}=$ $1 / 2+i \varpi_{k}$, a pole at $s=1$ with different residues, and trivial zeros $s=-2 h$ with $h \in \mathbb{N}$. Its nontrivial zeros for $L(s, \chi)=L\left(s, \chi_{0}\right)$ lie on the critical line $s=1 / 2$ and in the critical trip $0<\operatorname{Re}(s)<1$.

As shown in Case 3 of Section 1, we have followings:

- When $\chi=\chi^{*}$ and $\chi^{*}(-1)=1$,

$$
L(s, \chi)=L\left(s, \chi^{*}\right)=(q / \pi)^{-\frac{s}{2}} \xi\left(s, \chi^{*}\right) / \Gamma(s / 2)
$$

is an integral function of order 1 , and has the zeros (all zeros are the nontrivial zeros) $\rho_{k}=1 / 2+i \beta_{k}$ with $k \in \mathbb{N}$.

- When $\chi=\chi^{*}$ and $\chi^{*}(-1)=-1$,

$$
L(s, \chi)=L\left(s, \chi^{*}\right)=(q / \pi)^{-\frac{s+1}{2}} \xi(s, \chi) / \Gamma(s / 2+1 / 2)
$$

is an integral function of order 1 , and has the zeros (all zeros are the nontrivial zeros) $\rho_{k}=1 / 2+i \beta_{k}$ with $k \in \mathbb{N}$. It is observed that in Case 3 of Section 
1 , they have the nontrivial zeros $\rho_{k}=1 / 2+i \beta_{k}$ with $k \in \mathbb{N}$, which lie on the critical line $s=1 / 2$ and in the critical trip $0<\operatorname{Re}(s)<1$.

\section{NeW RESUlts AND APplications}

In this section we report the new formulas associated with the Dirichlet $L$-functions. Then we also give the new representations for the Wang theorems. Main target of the part is to present the applications of Conjecture 1 and Hardy-Littlewood theorems to obtain the Goldbach's conjecture.

4.1. New formulas for the Dirichlet $L$-functions. We now give the properties for the Dirichlet $L$-functions.

Theorem 3. Suppose that $\chi^{*}$ is a primitive character modulo $q$ with $q>1$. Let $\beta_{k}$ be the Siegel zeros for the Dirichlet L-functions with the primitive character $\chi^{*}$ with $k \in \mathbb{N}$. Then there exist the equivalent representations:

$$
\xi\left(s, \chi^{*}\right)=\xi\left(0, \chi^{*}\right) e^{s B\left(\chi^{*}\right)} \prod_{k=1}^{\infty}\left(1-\frac{s}{\frac{1}{2}+i \beta_{k}}\right) e^{s /\left(1 / 2+i \beta_{k}\right)},
$$

and

$$
\xi\left(s, \chi^{*}\right)=\xi\left(\frac{1}{2}, \chi^{*}\right) e^{\left(s-\frac{1}{2}\right) B\left(\chi^{*}\right)} \prod_{k=1}^{\infty}\left(1-\frac{s-\frac{1}{2}}{i \beta_{k}}\right) e^{(s-1 / 2) /\left(1 / 2+i \beta_{k}\right)},
$$

where $s \in \mathbb{C}$, and

$$
B\left(\chi^{*}\right)=\frac{\xi^{(1)}}{\xi}\left(0, \chi^{*}\right) .
$$

Proof. By Conjecture 1 and Theorem 1, we give the desired results.

Theorem 4. Suppose that $\chi^{*}$ is a primitive character modulo $q$ with $q>1$ and $\beta_{k}$ run the Siegel zeros for the Dirichlet $L$-functions with the primitive character $\chi^{*}$ with $k \in \mathbb{N}$. Then there exist the equivalent representations:

$$
L\left(s, \chi^{*}\right)=\frac{\xi\left(0, \chi^{*}\right) \cdot\left(\frac{q}{\pi}\right)^{-\frac{s+\hbar\left(\chi^{*}\right)}{2}} \cdot e^{B\left(\chi^{*}\right) s}}{\Gamma\left(\frac{s+\hbar\left(\chi^{*}\right)}{2}\right)} \cdot \prod_{k=1}^{\infty}\left(1-\frac{s}{\frac{1}{2}+i \beta_{k}}\right) e^{s /\left(1 / 2+i \beta_{k}\right)}
$$

and

$$
L\left(s, \chi^{*}\right)=\frac{\xi\left(\frac{1}{2}, \chi^{*}\right) \cdot\left(\frac{q}{\pi}\right)^{-\frac{s+\hbar\left(\chi^{*}\right)}{2}} \cdot e^{\left(s-\frac{1}{2}\right) B\left(\chi^{*}\right)}}{\Gamma\left(\frac{s+\hbar\left(\chi^{*}\right)}{2}\right)} \cdot \prod_{k=1}^{\infty}\left(1-\frac{s-\frac{1}{2}}{i \beta_{k}}\right) e^{(s-1 / 2) /\left(1 / 2+i \beta_{k}\right)}
$$


where $s \in \mathbb{C}, \hbar\left(\chi^{*}\right)$ is defined in Eq. (9), and

$$
B\left(\chi^{*}\right)=\frac{\xi^{(1)}}{\xi}\left(0, \chi^{*}\right) \text {. }
$$

Proof. By Conjecture 1 and Theorem 2, we give the desired results.

Theorem 5. Suppose that $\chi^{*}$ is a primitive character modulo $q$ with $q>1$ and $\beta_{k}$ are the Siegel zeros. Then there exist the equivalent representations:

$$
\Xi\left(\beta, \chi^{*}\right)=\xi\left(0, \chi^{*}\right) e^{(1 / 2+i \beta) B\left(\chi^{*}\right)} \prod_{k=1}^{\infty}\left(1-\frac{\frac{1}{2}+i \beta}{\frac{1}{2}+i \beta_{k}}\right) e^{(1 / 2+i \beta) /\left(1 / 2+i \beta_{k}\right)},
$$

and

$$
\Xi\left(\beta, \chi^{*}\right)=\xi\left(\frac{1}{2}, \chi^{*}\right) e^{i \beta B\left(\chi^{*}\right)} \prod_{k=1}^{\infty}\left(1-\frac{\beta}{\beta_{k}}\right) e^{i \beta /\left(1 / 2+i \beta_{k}\right)},
$$

where $\beta \in \mathbb{C}, \hbar\left(\chi^{*}\right)$ is defined in $E q .(9)$, and

$$
B\left(\chi^{*}\right)=\frac{\xi^{(1)}}{\xi}\left(0, \chi^{*}\right)
$$

Proof. By Theorem 3 and Conjecture 1, we obtain the desired results.

We now define the function by

$$
\xi\left(s, \chi_{0}\right)=\Re(s) L\left(s, \chi_{0}\right)=(s-1) \pi^{-s / 2} \Gamma(s / 2+1) L\left(s, \chi_{0}\right),
$$

where $s \in \mathbb{C}$ and $s \neq 1$.

Remark. Suppose that $\chi_{0}$ is the principal character modulo $q$ with $q>1$. Then we have that

$$
\xi\left(s, \chi_{0}\right)=\xi(0) e^{s \Im_{0}} \prod_{k=1}^{\infty}\left(1-\frac{s}{s_{k}}\right) e^{s / s_{k}} \prod_{p \mid q}\left(1-\frac{1}{p^{s}}\right)
$$

and

$$
\xi\left(s, \chi_{0}\right)=\xi(1 / 2) e^{s \Im_{0}} \prod_{k=1}^{\infty}\left(1-\frac{s-\frac{1}{2}}{s_{k}-\frac{1}{2}}\right) e^{s / s_{k}} \prod_{p \mid q}\left(1-\frac{1}{p^{s}}\right),
$$

where $s \in \mathbb{C}$ and $s \neq 1$.

Because Conjecture 1 is true, $1-\rho_{k}, \overline{\rho_{k}}$ and $1-\overline{\rho_{k}}$ are the nontrivial zeros for the Dirichlet $L$-functions, which is in agreement with the results of Montgomery and Vaughan [3]. 
From Eq. (152), we have

$$
\Xi\left(\beta, \chi^{*}\right)=\xi\left(\frac{1}{2}, \chi^{*}\right) e^{i \beta B\left(\chi^{*}\right)} \prod_{k=1}^{\infty}\left(1-\frac{\beta}{\beta_{k}}\right) e^{i \beta /\left(1 / 2+i \beta_{k}\right)},
$$

and

$$
\Xi\left(-\beta, \chi^{*}\right)=\xi\left(\frac{1}{2}, \chi^{*}\right) e^{-i \beta B\left(\chi^{*}\right)} \prod_{k=1}^{\infty}\left(1+\frac{\beta}{\beta_{k}}\right) e^{-i \beta /\left(1 / 2+i \beta_{k}\right)},
$$

such that

$$
\Xi\left(\beta, \chi^{*}\right) \Xi\left(-\beta, \chi^{*}\right)=\xi^{2}\left(\frac{1}{2}, \chi^{*}\right) \prod_{k=1}^{\infty}\left(1-\frac{\beta^{2}}{\beta_{k}^{2}}\right) .
$$

Let

$$
\rho_{k}^{*}=\operatorname{Re}\left(\rho_{k}\right)+i\left|\operatorname{Im}\left(\rho_{k}\right)\right|
$$

such that $1-\rho_{k}$ are the nontrivial zeros for the Dirichlet $L$-functions.

By Lemma 8 and Eq. (160), we have

$$
\begin{aligned}
& \xi\left(s, \chi^{*}\right) \\
& =\xi\left(0, \chi^{*}\right) e^{B\left(\chi^{*}\right) s} \prod_{k=1}^{\infty}\left(1-\frac{s}{\rho_{k}^{*}}\right) e^{s / \rho_{k}^{*}} \\
& =\xi\left(\frac{1}{2}, \chi^{*}\right) e^{(s-1 / 2) B\left(\chi^{*}\right)} \prod_{k=1}^{\infty}\left(1-\frac{s-\frac{1}{2}}{\rho_{k}^{*}-\frac{1}{2}}\right) e^{(s-1 / 2) / \rho_{k}^{*}}
\end{aligned}
$$

and

$$
\begin{aligned}
& \xi\left(s, \chi^{*}\right) \\
& =\xi\left(0, \chi^{*}\right) e^{B\left(\chi^{*}\right) s} \prod_{k=1}^{\infty}\left(1-\frac{s}{1-\rho_{k}^{*}}\right) e^{s /\left(1-\rho_{k}^{*}\right)} \\
& =\xi\left(\frac{1}{2}, \chi^{*}\right) e^{(s-1 / 2) B\left(\chi^{*}\right)} \prod_{k=1}^{\infty}\left(1+\frac{s-\frac{1}{2}}{\rho_{k}^{*}-\frac{1}{2}}\right) e^{(s-1 / 2) /\left(1-\rho_{k}^{*}\right)}
\end{aligned}
$$

such that

$$
\begin{aligned}
& \xi^{2}\left(s, \chi^{*}\right) \\
& =\xi^{2}\left(0, \chi^{*}\right) e^{2 s B\left(\chi^{*}\right)} \prod_{k=1}^{\infty}\left(1-\frac{s}{\rho_{k}^{*}}\right) \prod_{k=1}^{\infty}\left(1-\frac{s}{1-\rho_{k}^{*}}\right) e^{\frac{s}{\rho_{k}^{*}\left(1-\frac{*}{k}\right)}} \\
& =\xi^{2}\left(\frac{1}{2}, \chi^{*}\right) e^{2(s-1 / 2) B\left(\chi^{*}\right)} \prod_{k=1}^{\infty}\left(1-\left(\frac{s-\frac{1}{2}}{\rho_{k}^{*}-\frac{1}{2}}\right)^{2}\right) e^{\frac{s-1 / 2}{\rho_{k}^{*}\left(1-\rho_{k}^{*}\right)}} .
\end{aligned}
$$

According to Lemma 11 and Conjecture 1, we set up the following result. 
Corollary 1. Let $\chi(n)$ be a Dirichlet character $(\bmod q>1)$. Suppose that $N(T, \chi)$ denotes the number of zeros of the Dirichlet $L$-function (1) for $\operatorname{Re}(s)=1 / 2, T>2$ and $0 \leq \operatorname{Im}(s) \leq T$ and $\tilde{N}(T, \chi)$ denotes the number of zeros of the Dirichlet $L$-function (1) for $0<\operatorname{Re}(s)<1, T>2$ and $0 \leq \operatorname{Im}(s) \leq T$. Then we have

$$
N(T, \chi)=\tilde{N}(T, \chi) \text {. }
$$

Proof. From the obtained results in Section 3, we find the followings:

When $\chi=\chi_{0}$ and $q=1$, Eq. (164) is true (see [8,9] for further details), where

$$
L(s, \chi)=\zeta(s) \text {. }
$$

When $\chi=\chi_{0}$ and $q>1$, we have

$$
N\left(T, \chi_{0}\right)=\widetilde{N}\left(T, \chi_{0}\right)
$$

where $L(s, \chi)=L\left(s, \chi_{0}\right)$.

When $\chi=\chi^{*}$ and $\chi^{*}(-1)=1$, we conclude that

$$
N\left(T, \chi^{*}\right)=\widetilde{N}\left(T, \chi^{*}\right) .
$$

where $L(s, \chi)=L\left(s, \chi^{*}\right)=(q / \pi)^{-\frac{s}{2}} \xi\left(s, \chi^{*}\right) / \Gamma(s / 2)$.

When $\chi=\chi^{*}$ and $\chi^{*}(-1)=-1$, we arrive at

$$
N\left(T, \chi^{*}\right)=\tilde{N}\left(T, \chi^{*}\right)
$$

where $L(s, \chi)=L\left(s, \chi^{*}\right)=(q / \pi)^{-\frac{s+1}{2}} \xi(s, \chi) / \Gamma(s / 2+1 / 2)$.

Thus, we finish the proof.

4.2. The truth of the Goldbach's conjecture. We now give new representations for the Wang theorems and present the applications of the Hardy-Littlewood theorems to the Goldbach's conjecture because Conjecture 1 is proved and true.

Theorem 6. (The weak Goldbach's conjecture)

Every odd number $m>5$ is the sum of three primes.

Proof. By Lemma 13, we have show the required result since Conjecture 1 is true.

Theorem 7. (Wang Theorem I)

$(1,4)$ is valid, where $\delta_{2} \geq 3.237 / 2.237$.

Proof. By using Lemma 14 and considering the fact Conjecture 1 is proved and true, we get the result.

Theorem 8. (Wang Theorem II)

$(1,3)$ is valid, where $\delta_{1} \geq 2.475 / 1.475$. 
Proof. Similarly, by using Lemma 15, we have the result because Conjecture 1 is true.

Theorem 9. (The strong Goldbach's conjecture)

Every even number $m>2$ is sums of two primes.

Proof. By Lemma 16, Theorem 9 is true because of true of Conjecture 1.

Remark. By the work of Deshouillers and coauthors [15], we have that that the weak Goldbach's conjecture is true because Conjecture 1 is valid. By the work of Granville [18], we also see that the strong Goldbach's conjecture is true because Conjecture 1 is valid. Thus, the Goldbach's conjecture is true.

\section{Conclusion}

The present paper has proved that Conjecture 1 is true with the variant of the product for the entire function related to the Dirichlet $L$-functions. We have presented the applications of it to the Wang theorems for the Goldbach's problems. By using the Hardy-Littlewood theorems, we have shown that every odd number $m>5$ is the sum of three primes and that every even number $m>2$ is sums of two primes. The obtained result is proposed to solve the mathematical problems under the assumption of the truth of Conjecture 1.

Acknowledgements. This work is supported by the Yue-Qi Scholar of the China University of Mining and Technology (No. 102504180004).

\section{REFERENCES}

[1] Dirichlet, P. L. (1837). Beweis des Satzes, dass jede unbegrenzte arithmetische Progression, deren erstes Glied und Differenz ganze Zahlen ohne gemeinschaftlichen Factor sind, unendlich viele Primzahlen enthält. Abhandlungen der Königlich Preussischen Akademie der Wissenschaften, 1837(8), 45-81; (Euvres complètes, 1, 313-342.

[2] Davenport, H. (1980). Multiplicative number theory. Springer.

[3] Montgomery, H. L., Vaughan, R. C. (2007). Multiplicative number theory I: Classical theory. Cambridge university press.

[4] Riemann, G. F. B. (1859). Über die Anzahl der Primzahlen unter einer gegebenen Grösse. Monatsberichte der Deutschen Akademie der Wissenschaften zu Berlin, 2, 671-680.

[5] Yang, X. J. (2021). An introduction to hypergeometric, supertrigonometric, and superhyperbolic functions. Academic Press.

[6] Edwards, H. M. (1974). Riemanns'Zeta Function. Academic press, New York.

[7] Heath-Brown, D. (2005). Prime number theory and the Riemann zeta-function. In F. Mezzadri \& N. Snaith (Eds.), Recent Perspectives in Random Matrix Theory and Number Theory (London Mathematical Society Lecture Note Series, pp. 1-30). Cambridge: Cambridge University Press.

[8] Yang, X. J. (2021). Analysis of the Riemann zeta function. Reprint (1811.02418v10). 
[9] Yang, X. J. (2021). New infinite product formulas for the Riemann zeta-function applied to prove the Riemann conjecture. Reprint (1811.02418v7).

[10] Siegel, C. L. (1932). Uber Riemann's Nachlass zur analytischen Zahlentheorie. Quellen und Studien zur Geschichte der Mathematik, Astronomie und Physik, 2 (1932), 45-80 (Reprinted in C. L. Siegel, Gesammelte Abhandlungen, vol. 1, Springer, 1966, pp. 275-310).

[11] Landau, E. (1909). Handbuch der Lehre von der Verteilung der Primzahlen, Teubner, Leipzig.

[12] Siegel, C. L. (1943). Contributions to the theory of the Dirichlet L-series and the Epstein zeta-functions. Annals of Mathematics, 44(2), 143-172.

[13] Suriajaya, A. I. (2017). Two estimates on the distribution of zeros of the first derivative of Dirichlet $L$-functions under the generalized Riemann hypothesis. Journal de Théorie des Nombres de Bordeaux, 29(2), 471-502.

[14] Hardy, G. H., Littlewood, J. E. (1923). Some problems of "Partitio numerorum"; III: On the expression of a number as a sum of primes. Acta Mathematica, 44, 1-70.

[15] Deshouillers, J. M., Effinger, G., Te Riele, H., Zinoviev, D. (1997). A complete Vinogradov 3-primes theorem under the Riemann hypothesis. Electronic Research Announcements of the American Mathematical Society, 3, 99-104; In: Yuan Wang (Ed.), The Goldbach Conjecture (pp. 91-96), World Scientific, 2002.

[16] Yuan Wang. (1962). On the representation of large integer as a sum of a prime and an almost prime, Scientia Sinica, 12(8), 168-181; In: Yuan Wang (Ed.), The Goldbach Conjecture (pp. pp. 170-191), World Scientific, 1984.

[17] Hardy, G. H., Littlewood, J. E. (1923). Some problems of "Partitio Numerorum "(V): A further contribution to the study of Goldbach's problem. Proceedings of the London Mathematical Society, 2(1), 46-56.

[18] Granville, A. (2007). Refinements of Goldbach's conjecture, and the generalized Riemann hypothesis. Functiones et Approximatio Commentarii Mathematici, 37(1), 159-173.

[19] Oliveira e Silva, T., Herzog, S., Pardi, S. (2014). Empirical verification of the even Goldbach conjecture and computation of prime gaps up to $4 \cdot 10^{18}$. Mathematics of Computation, $83(288)$, 2033-2060.

[20] Hadamard, J. (1893). Étude sur les propriétés des fonctions entières et en particulier d'une fonction considérée par Riemann. Journal de Mathématiques Pures et Appliquées, 171-216.

[21] Hadamard, J. (1896). Sur la distribution des zéros de la fonction $\zeta(s)$ et ses conséquences arithmétiques. Bulletin de la Societé Mathematique de France, 24, 199-220.

[22] Vallée-Poussin, C. D. L. de la. (1896). Recherches analytiques sur la théorie des nombres premiers, I-III. Annales de la Société scientifique de Bruxelles, 20, 183-256.

[23] Broughan, K. (2017). Equivalents of the Riemann Hypothesis: Volume 2, Analytic Equivalents. Cambridge University Press, London.

[24] Patterson, S. J. (1988). An introduction to the theory of the Riemann zeta-function. Cambridge University Press.

[25] Landau, E. (1915). Über die Hardysche Entdeckung unendlich vieler Nullstellen der Zetafunktion mit reellem Teil 1/2. Mathematische Annalen, 76(2), 212-243.

[26] Helfgott, H. A. (2013). The ternary Goldbach conjecture is true. Preprint (1312.7748).

[27] Helfgott, H. A. (2013). The ternary Goldbach conjecture. La Gaceta de la Real Sociedad Matematica Espanola, 16(4), 709-726.

[28] Chen, J. R. (1973). on the representation of a larger even integer as the sum of a prime and the product of at most two primes, Scientia Sinica, 3(2),111-128. 
[29] Chen, J. R. (1978). On the representation of a large even integer as the sum of a prime and the product of at most two primes (II). Scientia Sinica, 21(4), 421-430.

[30] Helfgott, H. A., Platt, D. J. (2013). Numerical verification of the ternary Goldbach conjecture up to $8.875 \cdot 10^{30}$. Experimental Mathematics, 22(4), 406-409.

Davis, S. (2019). Nontrivial zeros of $L$-functions. Journal of Interdisciplinary Mathematics, $22(5), 621 \mathrm{C} 636$.

E-mail address: dyangxiaojun@163.com; xjyang@cumt.edu.cn

1 School of Mathematics, China University of Mining and Technology, Xuzhou 221116, CHINA

2 State Key Laboratory for Geomechanics and Deep Underground Engineering, China University of Mining and Technology, Xuzhou 221116, China

3 School of Mechanics and Civil Engineering, China University of Mining and Technology, Xuzhou 221116, China 